\title{
The Net in a Fuzzy Soft Topological Space and Its Applications
}

\author{
Rui Gao $(1)$ and Jianrong $W u$ \\ College of Mathematics Science, Suzhou University of Science and Technology, Suzhou, Jiangsu 215009, China \\ Correspondence should be addressed to Jianrong Wu; jrwu@mail.usts.edu.cn
}

Received 23 November 2020; Revised 13 February 2021; Accepted 24 March 2021; Published 7 April 2021

Academic Editor: Feng Feng

Copyright (c) 2021 Rui Gao and Jianrong Wu. This is an open access article distributed under the Creative Commons Attribution License, which permits unrestricted use, distribution, and reproduction in any medium, provided the original work is properly cited.

In this paper, the fuzzy soft net is explored to study the properties of a fuzzy soft topological space. Some important results about the closure, separation, and compactness are obtained. It is demonstrated that the net is a powerful tool for studying fuzzy soft topological spaces.

\section{Introduction}

In 2001, Maji et al. [1] combined fuzzy sets [2] with soft sets [3] and proposed the concept of fuzzy soft sets. After that, the fuzzy soft set was applied to group theory, decision-making, medical diagnosis, and other fields (see [4-13]). Meanwhile, the theory of fuzzy soft set has been developed rapidly. In particular, the research on fuzzy soft topology has made a lot of achievements (see [14-24]).

Noting the contribution of the pointed approach in fuzzy topology, Roy and Samanta [21] defined a fuzzy soft point on a fuzzy soft topological space. In 2018, Ibedou and Abbas [17] redefined this concept. Recently, Gao and $\mathrm{Wu}$ [7] studied the properties of fuzzy soft points introduced in [17] deeply and pointed that the fuzzy soft point given in [17] was more effective than that given in [21]. They also gave the definitions of a fuzzy soft net consisting of fuzzy soft points and its convergence. On these bases, they characterized the continuity of fuzzy soft mappings by the net approach.

This paper aims to further the study of [7]. In Section 2, some preliminaries will be recalled. In Section 3, the characterizations of some important results involving closure, separation, and compactness will be obtained by means of fuzzy soft nets.

\section{Preliminaries}

Throughout this paper, $U$ refers to an initial universe and $E$ is the set of all parameters for $U$. In this case, $U$ is also denoted by $(U, E) . I^{U}$ is the set of all fuzzy subsets over $U$, where $I=[0,1]$. The elements $\overline{0}, \overline{1} \in I^{U}$, respectively, refer to the functions $\overline{0}(x)=0$ and $\overline{1}(x)=1$ for all $x \in U$. For an element $A \in I^{U}$, if there exists an $x \in U$ such that $A(x)=$ $\lambda>0$ and $A(y)=0, \forall y \in(U /\{x\})$, then $A$ is called a fuzzy point over $U$ and is denoted by $x_{\lambda}, x$ and $\lambda$ are its support and height, respectively.

The definitions in this section are all sourced from the existing literature $[7,17,21,22]$.

Definition 1. Let $A \subseteq E$. A mapping $F_{A}: E \longrightarrow I^{U}$, is called a fuzzy soft set over $(U, E)$, where $F_{A}(e)=\overline{0}$ if $e \in(E / A)$ and $F_{A}(e) \neq \overline{0}$ if $e \in A$.

The set of all fuzzy soft sets over $(U, E)$ is denoted by $\mathrm{FS}(U, E)$.

The fuzzy soft set $F_{\phi} \in \mathrm{FS}(U, E)$ is called the null fuzzy soft set and is denoted by $\widetilde{\Phi}$. Here, $F_{\phi}(e)=\overline{0}$ for every $e \in E$.

For $F_{E} \in \mathrm{FS}(U, E)$, if $F_{E}(e)=\overline{1}$ for all $e \in E$, then $F_{E}$ is called the absolute fuzzy soft set and is denoted by $\widetilde{E}$. 
Let $F_{A}, F_{B} \in \mathrm{FS}(U, E)$. If $F_{A}(e) \subseteq F_{B}(e)$ for all $e \in E$, then $F_{A}$ is said to be a fuzzy soft subset of $F_{B}$ and is denoted by $F_{A} \widetilde{\subseteq} F_{B}$ or $F_{B} \supseteq F_{A}$. In addition, $F_{A} \widetilde{\subseteq} F_{B}$ means that $F_{A}$ is not a fuzzy soft subset of $F_{B}$. If $F_{A} \widetilde{\simeq} F_{B}$ and $F_{B} \widetilde{\subseteq} F_{A}$, then $F_{A}$ and $F_{B}$ are said to be equivalent, which is denoted by $F_{A}=F_{B}$.

Remark 1. If $F_{A} \widetilde{\subseteq} F_{B}$, then $A \subseteq B$.

Definition 2. Let $F_{A}, F_{B} \in \mathrm{FS}(U, E)$.

(1) The complement of $F_{A}$, denoted by $F_{A}^{c}$, is then defined as

$$
F_{A}^{c}(e)= \begin{cases}\overline{1}-F_{A}(e) & \text { for } e \in A, \\ \overline{1}, & \text { otherwise }\end{cases}
$$

(2) The union of $F_{A}$ and $F_{B}$ is also a fuzzy soft set $F_{C}$ defined by $F_{C}(e)=F_{A}(e) \cup F_{B}(e)$ for all $e \in E$, where $C=A \cup B$, and is denoted by $F_{C}=F_{A} \cup F_{B}$.

(3) The intersection of $F_{A}$ and $F_{B}$ is also a fuzzy soft set $F_{C}$ defined by $F_{C}(e)=F_{A}(e) \cap F_{B}(e)$ for all $e \in E$, where $C=A \cap B$, and is denoted by $F_{C}=F_{A} \widetilde{\cap} F_{B}$.

Similarly, the union (intersection) of a family of fuzzy soft sets may be defined as $\left\{F_{C_{\alpha}}: \alpha \in \Lambda\right\}$ and denoted by $\widetilde{\cup}_{\alpha \in \Lambda} F_{C_{\alpha}}\left(\widetilde{\cap}_{\alpha \in \Lambda} F_{C_{\alpha}}\right)$, where $\Lambda$ is an arbitrary index set.

Remark 2. The following is, therefore, clear:

(1) $\widetilde{\Phi}^{c}=\widetilde{E}, \widetilde{E}^{c}=\widetilde{\Phi}$

(2) $\left(\widetilde{\cup}_{\alpha \in \Lambda} F_{A_{\alpha}}\right)^{c}=\widetilde{\cap}_{\alpha \in \Lambda} F_{A_{\alpha}}^{c}$

(3) $\left(\widetilde{\cap}_{\alpha \in \Lambda} F_{A_{\alpha}}\right)^{c}=\widetilde{U}_{\alpha \in \Lambda} F_{A_{\alpha}}^{c}$

Definition 3. A fuzzy soft topology $\tau$ over $(U, E)$ is a family of fuzzy soft sets over $(U, E)$ satisfying the following properties:

(1) $\widetilde{\Phi}, \widetilde{E} \in \tau$

(2) If $F_{A}, F_{B} \in \tau$, then $F_{A} \widetilde{\cap} F_{B} \in \tau$

(3) If $F_{A_{\alpha}} \in \tau$ for all $\alpha \in \Lambda$ (an index set), then

If $\tau$ is a fuzzy soft topology over $(U, E)$, the triple $(U, E, \tau)$ is said to be a fuzzy soft topological space. Each element of $\tau$ is called an open set. If $F_{A}^{c}$ is an open set, then $F_{A}$ is called a closed set.

Definition 4. Let $(U, E, \tau)$ be a fuzzy soft topological space, $F_{A} \in \mathrm{FS}(U, E)$.

(1) The intersection of all closed sets $F_{B} \supseteq F_{A}$ is called the closure of $F_{A}$ and is denoted by $\frac{B}{F_{A}}$

(2) The union of all open subsets of $F_{A}$ over $(U, E, \tau)$ is called the interior of $F_{A}$ and is denoted by $\operatorname{int} F_{A}$

Remark 3. Let $F_{A} \in \mathrm{FS}(U, E)$. The following is evident:

(1) $\overline{F_{A}}$ is closed and $\operatorname{int} F_{A}$ is open

(2) $F_{A}$ is closed if and only if $F_{A}=\overline{F_{A}}$
(3) $F_{A}$ is open if and only if and $F_{A}=\operatorname{int} F_{A}$

Definition 5. A mapping $\xi: E \longrightarrow I^{U}$ is called a fuzzy soft point over $(U, E)$ if there is an $e \in E$ such that $\xi(e)=x_{\lambda} \in \mathrm{FP}(U)$, and $\xi(a)=\overline{0}$ when $a \in(E /\{e\})$.

In this case, $\xi$ is also denoted by $P_{e}^{x_{\lambda}}$, and $e$ is called its parameter support. The set of all fuzzy soft points over $(U, E)$ is denoted by $\operatorname{FSP}(U, E)$.

In the remainder of this paper, a fuzzy soft point is always referred to as given by Definition 5 and is called a point for short.

For $P_{e}^{x_{\lambda}}, P_{f}^{y_{\beta}} \in \operatorname{FSP}(U, E)$, if $x \neq y$ or $\lambda \neq \beta$ or $e \neq f$, then it is said that $P_{e}^{x_{\lambda}}$ and $P_{f}^{y_{\beta}}$ are different, which is written as $P_{e}^{x_{\lambda}} \neq P_{f}^{y_{\beta}}$.

Theorem 1. Let $P_{e}^{x_{\lambda}} \in F S P(U, E), F_{A}, F_{B} \in F S(U, E)$, and $F_{A_{\alpha}} \in F S(U, E)(\forall \alpha \in \Lambda)$.

(1) $P_{e}^{x_{\lambda}} \in F_{A} \widetilde{\cup} F_{B}$ if and only if $P_{e}^{x_{\lambda}} \in F_{A}$ or $P_{e}^{x_{\lambda}} \in F_{B}$

(2) $P_{e}^{x_{\lambda}} \in F_{A_{\alpha}}(\forall \alpha \in \Lambda)$ if and only if $P_{e}^{x_{\lambda}} \in \widetilde{\cap}_{\alpha \in \Lambda} F_{A_{\alpha}}$

Definition 6. A point $P_{e}^{x_{\lambda}}$ is said to be quasi-coincident with $F_{A} \in \mathrm{FS}(U, E)$, which is denoted by $P_{e}^{x_{\lambda}} \tilde{\in} F_{A}$, if $\lambda+F_{A}(e)(x)>1$.

That $P_{e}^{x_{\lambda}}$ is not quasi-coincident with $F_{A}$ is denoted by

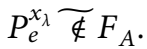

Remark 4. It is obvious that $P_{e}^{x_{\lambda}} \tilde{\epsilon} F_{A}$ if and only if $P_{e}^{x_{\lambda}} \notin F_{A}^{c}$. Additionally, if $P_{e}^{x_{\lambda}} \tilde{\in} F_{A}$, then there exists $0<\mu<\lambda$ such that $P_{e}^{x_{1-\mu}} \in F_{A}$.

Theorem 2. Let $F_{A}, F_{B}, F_{A_{\alpha}} \in F S(U, E) \quad(\forall \alpha \in \Lambda)$, and $P_{e}^{x_{\lambda}} \in F S P(U, E)$.

(1) $F_{A} \tilde{\subseteq} F_{B}$ if and only if $P_{e}^{x_{\lambda}} \tilde{\in} F_{B}$ for any $P_{e}^{x_{\lambda}} \tilde{\in} F_{A}$

(2) $P_{e}^{x_{\lambda}} \tilde{\in} \widetilde{\cap}_{\alpha \in \Lambda} F_{A_{\alpha}}$ implies $P_{e}^{x_{\lambda}} \tilde{\in} F_{A_{\alpha}}(\forall \alpha \in \Lambda)$

(3) $P_{e}^{x_{\lambda}} \tilde{\in} F_{A} \widetilde{\cap} F_{B}$ if and only if $P_{e}^{x_{\lambda}} \widetilde{\in} F_{A}$ and $P_{e}^{x_{\lambda}} \tilde{\in} F_{B}$

(4) $P_{e}^{x_{\lambda}} \tilde{\epsilon} \widetilde{U}_{\alpha \in \Lambda} F_{A_{\alpha}}$ if and only if there exists an $\alpha_{0} \in \Lambda$ such that $P_{e}^{x_{\lambda}} \stackrel{\underline{\alpha}}{\epsilon} F_{A_{\alpha_{0}}}$

Definition 7. Let $F_{A}, F_{B} \in \mathrm{FS}(U, E)$ and $\xi \in \mathrm{FSP}(U, E)$. If $\xi \widetilde{\epsilon} F_{A} \widetilde{\cap} F_{B}$, then it is said that $F_{A}$ and $F_{B}$ are quasi-coincident at $\xi$.

Definition 8. A fuzzy soft set $F_{A}$ is said to be quasi-coincident with $F_{B}$, which is denoted by $F_{A} q F_{B}$, if $F_{A}(e)(x)+$ $F_{B}(e)(x)>1$ for some $x \in U$ and $e \in A \cap B$.

That $F_{A}$ is not quasi-coincident with $F_{B}$ is denoted by $F_{A} \bar{q} F_{B}$.

Theorem 3. Let $F_{A}, F_{B} \in F S(U, E)$. If $F_{A} q F_{B}$, then $F_{A} \widetilde{\cap} F_{B} \neq \widetilde{\Phi}$.

Definition 9. Let $\xi \in \operatorname{FSP}(U, E)$ and $F_{A}, F_{B} \in \operatorname{FS}(U, E)$.

(1) $F_{A}$ is said to be a neighborhood of $\xi$ if there exists $F_{B} \in \tau$ such that $\xi \in F_{B} \simeq F_{A}$ 
(2) $F_{A}$ is called a Q-neighborhood of $\xi$ if there exists $F_{B} \in \tau$ such that $\xi \tilde{\epsilon} F_{B} \widetilde{\subseteq} F_{A}$

The set of all Q-neighborhoods of $\xi$ is denoted by $\mathrm{A}(\xi)$. In the remainder of this paper, $\Delta$ is a directed set with the partial order "८".

Definition 10. The mapping $S: \Delta \longrightarrow \operatorname{FSP}(U, E)$ is called a fuzzy soft net over $(U, E)$ and is denoted by $\{S(\delta), \delta \in \Delta\}$ or $S$ for simplicity.

In particular, if there exists $F_{A} \in \mathrm{FS}(U, E)$ such that $S(\delta) \in F_{A}$ for any $\delta \in \Delta$, then $S$ is said to be a fuzzy soft net in $F_{A}$, or a net for simplicity.

Definition 11. Let $F_{A} \in \mathrm{FS}(U, E)$ and $S=\{S(\delta), \delta \in \Delta\}$ be a net over $(U, E)$. If there exists $\delta_{0} \in \Delta$ such that $S(\delta) \tilde{\epsilon} F_{A}$ whenever $\delta_{0}<\delta$, then $S$ is said to be eventually quasi-coincident with $F_{A}$. If for each $\delta \in \Delta$ there exists $\delta_{0} \in \Delta$ with $\delta<\delta_{0}$ such that $S\left(\delta_{0}\right) \tilde{\epsilon} F_{A}$, then $S$ is said to be frequently quasi-coincident with $F_{A}$.

Definition 12. A net $\{S(\delta), \delta \in \Delta\}$ over $(U, E, \tau)$ is said to be convergent to a point $\xi$ if $S$ is eventually quasi-coincident with each Q-neighborhood of $\xi$. In this case, $\xi$ is called the limit of $S$ and is denoted by $\lim S(\delta)$.

\section{Main Results}

In this section, nets are applied to characterize the closure of a fuzzy soft set, $T_{2}$ separation, and compactness of a fuzzy soft topological space.

Obviously, if $P_{e}^{x_{\lambda}} \in \operatorname{FSP}(U, E)$, then $\mathrm{A}\left(P_{e}^{x_{\lambda}}\right)$ forms a directed set under the relation $\widetilde{\simeq}$.

Theorem 4. Let $(U, E, \tau)$ be a fuzzy soft topological space. A point $\xi \widetilde{\epsilon} \operatorname{int} F_{A}$ if and only if there exists an $F_{B} \in \mathrm{A}(\xi)$ such that $F_{B} \tilde{\simeq} F_{A}$.

Proof (Necessity). Let $\xi \tilde{\epsilon} \operatorname{int} F_{A}$. From Definition 4 and Theorem 2 (4), there exists an open set $F_{B} \widetilde{\simeq} F_{A}$ such that $\xi \tilde{\epsilon} F_{B}$. Because $F_{B}$ is open, $F_{B} \in \mathrm{A}(\xi)$.

(Sufficiency) Let $F_{B} \in \mathrm{A}(\xi)$ and $F_{B} \tilde{\simeq} F_{A}$. From Definition 9, there is $F_{C} \in \tau$ such that $\xi \tilde{\in} F_{C} \widetilde{\subseteq} F_{B}$. It is obvious that $F_{C} \widetilde{\widetilde{\tau}} \operatorname{int} F_{B} \tilde{\widetilde{\simeq}} \operatorname{int} F_{A}$. Then, $\xi \tilde{\epsilon} \operatorname{int} F_{A}$.

Theorem 5. A point $P_{e}^{x_{\lambda}} \in \overline{F_{A}}$ if and only if each Q-neighborhood of $P_{e}^{x_{\lambda}}$ is quasi-coincident with $F_{A}$.

Proof (Necessity). Let $P_{e}^{x_{\lambda}} \in \overline{F_{A}}, F_{B} \in \mathrm{A}\left(P_{e}^{x_{\lambda}}\right)$. Without loss of generality, it is supposed that $F_{B}$ is open. To complete the proof of necessity, it is sufficient to show $F_{B} q F_{A}$. In fact, if $F_{B} \bar{q} F_{A}$, then $F_{A}(d)(y)+F_{B}(d)(y) \leq 1$ for any $y \in U$ and $d \in E$. Thus, $F_{A} \widetilde{\widetilde{\subseteq}} F_{B}^{c}$. Note that $F_{B}^{c}$ is closed and $\overline{F_{A}} \widetilde{\subseteq} F_{B}^{c}$. So, $P_{e}^{x_{\lambda}} \in F_{B}^{c}$, which together with Remark 4 conflicts with $P_{e}^{x_{\lambda}} \tilde{\epsilon} F_{B}$. Hence, $F_{B} q F_{A}$.

(Sufficiency) Suppose each Q-neighborhood of $P_{e}^{x_{\lambda}}$ is quasi-coincident with $F_{A}$. If $P_{e}^{x_{\lambda}} \notin \overline{F_{A}}$, then $P_{e}^{x_{\lambda}} \tilde{\epsilon}{\overline{F_{A}}}^{c}$. Thus, ${ }_{F}^{{ }_{A} C} \in \mathrm{A}\left(P_{e}^{x_{\lambda}}\right)$. It follows from the supposition that $\bar{F}_{A}{ }^{c} q F_{A}$. So, there exist $y \in U$ and $d \in E$ such that

$$
{\overline{F_{A}}}^{c}(d)(y)+F_{A}(d)(y)>1 .
$$

Hence, $\quad \overline{F_{A}}(d)(y)<F_{A}(d)(y), \quad$ which is a contradiction.

Theorem 6. Let $F_{A} \in F S(U, E)$. Then, a point $P_{e}^{x_{\lambda}} \in \overline{F_{A}}$ if and only if there is a net $S=\{S(\delta): \delta \in \Delta\}$ in $F_{A}$ such that $\lim S(\delta)=P_{e}^{x_{\lambda}}$.

Proof (Necessity). Let $P_{e}^{x_{\lambda}} \in \overline{F_{A}}$. Take $\left\{\mathrm{A}\left(P_{e}^{x_{\lambda}}\right), \underline{\underline{\simeq}}\right\}$ as a directed set. For any $F_{B} \in \mathrm{A}\left(P_{e}^{x_{\lambda}}\right)$, by Theorem $5, F_{B} q F_{A}$. Then, there exist $d_{B} \in E$ and $y_{B} \in U$ such that

$$
F_{B}\left(d_{B}\right)\left(y_{B}\right)+F_{A}\left(d_{B}\right)\left(y_{B}\right)>1 .
$$

Let $\lambda_{B}=F_{A}\left(d_{B}\right)\left(y_{B}\right)$. Then, the points $P_{d_{B}}^{\left(y_{B}\right)_{B}} \in F_{A}$ and

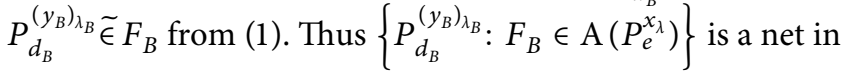
$F_{A}$.

It is now proven that $\left\{P_{d_{B}}^{\left(y_{B}\right)_{\lambda_{B}}}: F_{B} \in \mathrm{A}\left(P_{e}^{x_{\lambda}}\right)\right\}$ converges to $P_{e}^{x_{\lambda}}$. In fact, for any $F_{C} \in \mathrm{A}\left(P_{e}^{x_{\lambda}}\right)$, let $F_{B_{0}}=F_{C}$. When $F_{B_{0}} \simeq F_{B} \in \mathrm{A}\left(P_{e}^{x_{\lambda}}\right)$, by (3), the following is obtained:

$$
\begin{aligned}
1 & <F_{B}\left(d_{B}\right)\left(y_{B}\right)+F_{A}\left(d_{B}\right)\left(y_{B}\right) \\
& \leq F_{B_{0}}\left(d_{B}\right)\left(y_{B}\right)+\lambda_{B} .
\end{aligned}
$$

So, $P_{d_{B}}^{\left(y_{B}\right)_{\lambda_{B}}} \tilde{\in} F_{B_{0}}$, that is, $P_{d_{B}}^{\left(y_{B}\right)_{\lambda_{B}}} \tilde{\in} F_{C}$. From the arbitrar-

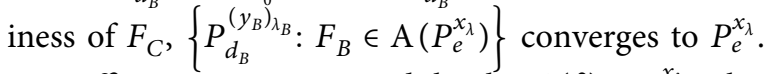

Sufficiency: it is supposed that $\lim S(\delta)=P_{e}^{x_{\lambda}}$, where $S=$ $\{S(\delta): \delta \in \Delta\}$ is in $F_{A}$. Then, for any $F_{B} \in \mathrm{A}\left(P_{e}^{x_{\lambda}}\right)$, there is a $\delta_{0} \in \Delta$ such that $S\left(\delta_{0}\right) \in F_{A}$ and $S\left(\delta_{0}\right) \tilde{\epsilon} F_{B} . S\left(\delta_{0}\right)$ is written as $P_{d}^{y_{B}}$, where $d \in E, \quad y \in U$, and $\beta \in(0,1]$. Thus $F_{A}(d)(y) \geq \beta \quad$ and $\quad \beta+F_{B}(d)(y)>1$. So, $F_{A}(d)(y)+F_{B}(d)(y)>1$, which means that $F_{B} q F_{A}$. From the arbitrariness of $F_{B}$ and Theorem $5, P_{e}^{x_{\lambda}} \in \overline{F_{A}}$ is obtained.

Definition 13. Let $(U, E, \tau)$ be a fuzzy soft topological space. If for any two different points $P_{e}^{x_{\lambda}}$ and $P_{f}^{y_{\beta}}$ there exist $F_{A} \in \mathrm{A}\left(P_{e}^{x_{\lambda}}\right)$ and $F_{B} \in \mathrm{A}\left(P_{f}^{y_{\beta}}\right)$ such that $F_{A} \cap F_{B}=\widetilde{\Phi}$, then $(U, E, \tau)$ is said to be $T_{2}$ separated.

Theorem 7. A fuzzy soft topological space $(U, E, \tau)$ is $T_{2}$ separated if and only if the limit of any net is unique.

Proof (Necessity). Suppose that a net $S=\{S(\lambda): \lambda \in \Lambda\}$ converges to two different points $P_{e}^{x_{\alpha}}$ and $P_{f}^{y_{\beta}}$. Because $(U, E, \tau)$ is $T_{2}$ separated, there exist $F_{A} \in A\left(P_{e}^{x_{\alpha}}\right)$ and $F_{B} \in \mathrm{A}\left(P_{f}^{y_{\beta}}\right)$ such that $F_{A} \widetilde{\cap} F_{B}=\widetilde{\Phi}$. By Definition 12, there exists $S(\lambda)$ such that $S(\lambda) \tilde{\epsilon} F_{A}$ and $S(\lambda) \tilde{\epsilon} F_{B}$. Then, $S(\lambda) \tilde{\epsilon} F_{A} \cap F_{B}$. There is a contradiction with the fact that $F_{A} \widetilde{\cap} F_{B}=\widetilde{\Phi}$.

Sufficiency: suppose that $(U, E, \tau)$ is not $T_{2}$ separated, then there are two different points $P_{e}^{x_{\alpha}}$ and $P_{f}^{y_{\beta}}$, and for any $F_{A} \in \mathrm{A}\left(P_{e}^{x_{\alpha}}\right)$ and $F_{B} \in \mathrm{A}\left(P_{f}^{y_{\beta}}\right), F_{A}$ and $F_{B}$ are quasi-coincident at some points. Thus, $S(A, B) \in \operatorname{FSP}(U, E)$ such that $S(A, B) \tilde{\epsilon} F_{A}$ and $S(A, B) \tilde{\epsilon} F_{B}$. Let $\Delta=\left\{\left(F_{A}, F_{B}\right)\right.$ : $\left.F_{A} \in \mathrm{A}\left(P_{e}^{x_{\alpha}}\right), F_{B} \in \mathrm{A}\left(P_{f}^{y_{\beta}}\right)\right\}$. Then, $\Delta$ forms a directed set 
under the order relation " $\prec$ " defined as $\left(F_{A}, F_{B}\right) \prec\left(F_{C}, F_{D}\right)$ if and only if $F_{A} \supseteq F_{C}, F_{B} \supseteq F_{D}$. For any $\delta=\left(F_{A}, F_{B}\right) \in \Delta$, let $S(\delta)=S(A, B)$. So, a net $S=\{S(\delta): \delta \in \Delta\}$ is obtained.

It is now shown that $S$ converges to $P_{e}^{x_{\alpha}}$ and $P_{f}^{y_{\beta}}$. In fact, for any $F_{A} \in \mathrm{A}\left(P_{e}^{x_{\alpha}}\right)$ and $F_{B} \in \mathrm{A}\left(P_{f}^{y_{\beta}}\right),\left(F_{A}, F_{B}\right) \in \Delta$. When $\left(F_{A}, F_{B}\right) \prec\left(F_{C}, F_{D}\right) \in \Delta, S(C, D) \tilde{\epsilon} F_{C}, S(C, D) \tilde{\epsilon} F_{D}, F_{A} \supseteq F_{C}$, and $F_{B} \widetilde{\supseteq} F_{D}$. Then, $S(C, D) \tilde{\epsilon} F_{A}$ and $S(C, D) \tilde{\epsilon} F_{B}$. From the arbitrariness of $F_{A}$ and $F_{B}, \lim S(\delta)=P_{e}^{x_{\alpha}}$ and $\lim S(\delta)=$ $P_{f}^{y_{\beta}}$ are obtained, which is a contradiction with the supposition.

A fuzzy soft net $\{T(\omega): \omega \in \Omega\}$ is called a subnet of a fuzzy soft net $\{S(\lambda): \lambda \in \Lambda\}$ if there exists a mapping $\varphi: \Omega \longrightarrow \Lambda$ such that

(i) $\forall p \in \Lambda$, there exists $\omega \in \Omega$ such that $p<\varphi(\omega)$

(ii) For any $\omega_{1}, \omega_{2} \in \Omega$ with $\omega_{1} \prec \omega_{2}$, then $\varphi\left(\omega_{1}\right) \prec \varphi\left(\omega_{2}\right)$

(iii) $T(\omega)=S(\varphi(\omega))$

Let $\Sigma$ be a family of fuzzy soft sets, and $F_{B} \in F S(U, E)$. If $F_{B} \underset{\subset}{ } \widetilde{U}_{F_{A} \in \Sigma} F_{A}$, then $\Sigma$ is called a cover of $F_{B}$. If a cover contains finite elements, then it is called a finite cover. If a subset $\Sigma_{1}$ of $\Sigma$ is also a cover of $F_{B}$, then $\Sigma_{1}$ is called a subcover of $\Sigma$. If $\Sigma$ is a cover of $F_{B}$, every element of $\Sigma$ being open (closed), then $\Sigma$ is said to be an open (closed) cover of $F_{B}$.

Definition 14. Let $F_{A} \in \mathrm{FS}(U, E)$. If every open cover of $F_{A}$ contains a finite subcover, then $F_{A}$ is said to be compact.

Lemma 1. Let $\xi \in F S P(U, E), \quad F_{A} \in F S(U, E)$, and $S=\{S(\lambda): \lambda \in \Lambda\}$ be a net. If any subnet of $S$ does not take $\xi$ as its limit, then there exists $O_{\xi} \in \mathrm{A}(\xi)$ and $\lambda_{\xi} \in \Lambda$ such that $S(\lambda) \approx O_{\xi}$ whenever $\lambda_{\xi} \prec \lambda \in \Lambda$.

Proof. Conversely, it is supposed that, for any $O \in \mathrm{A}(\xi)$ and any $\lambda \in \Lambda$, there exists $\lambda_{O} \in \Lambda$ with $\lambda \prec \lambda_{O}$ such that $S\left(\lambda_{O}\right) \tilde{\epsilon} O$. Let $\bar{\Lambda}$ be a set of the corresponding $\lambda_{O}$ with respect to all $O \in \mathrm{A}(\xi)$ and all $\lambda \in \Lambda$, that is, $\bar{\Lambda}=\left\{\lambda_{O}: \lambda<\lambda_{O}\right.$ and $\left.S\left(\lambda_{O}\right) \tilde{\epsilon} O, O \in \mathrm{A}(\xi), \lambda \in \Lambda\right\}$. Then, $\bar{\Lambda}$ is a directed set under the same order relation "२" as in $\Lambda$.

To complete the proof, it must only be shown that there is a subnet of $S$ converging to $\xi$. Set $\Omega=\mathrm{A}(\xi)$. Under the relation $\tilde{\supseteq}, \Omega$ is a directed set. Let $\Omega \times \bar{\Lambda}$ be the usual product directed set of $\Omega$ and $\bar{\Lambda}$. The mapping $\varphi$ from $\Omega \times \bar{\Lambda}$ to $\Lambda$ is defined as $\varphi\left(O, \lambda_{0}\right)=\lambda_{0}, \forall\left(O, \lambda_{O}\right) \in \Omega \times \bar{\Lambda}$. Then, for any $\lambda^{\prime} \in \Lambda$, there always exists $\left(O^{\prime}, \lambda_{O^{\prime}}\right) \in \Omega \times \bar{\Lambda}$ such that $\lambda \prime<\lambda_{O^{\prime}}$ and $S\left(\lambda_{O^{\prime}}\right) \widetilde{\epsilon} O^{\prime}$. When $\left(O^{\prime}, \lambda_{O^{\prime}}\right) \prec\left(O, \lambda_{O}\right) \in \Omega \times \bar{\Lambda}$, $\mathrm{O}^{\prime} \supseteq 0, \quad \lambda_{O^{\prime}} \prec \lambda_{O} . \quad$ Thus $\quad \lambda^{\prime} \prec \lambda_{O^{\prime}} \prec \lambda_{O}=\varphi\left(O, \lambda_{O}\right)$. So, $\left\{S\left(\lambda_{O}\right):\left(O, \lambda_{O}\right) \in \Omega \times \bar{\Lambda}\right\}$ is a subnet of $S=\{S(\lambda): \lambda \in \Lambda\}$. Let $O^{\prime} \in A(\xi)$ arbitrarily. Then, $\left(O, \lambda_{O^{\prime}}\right) \in \Omega \times \bar{\Lambda}$. When $\left(O^{\prime}, \lambda_{O^{\prime}}\right) \prec\left(O, \lambda_{O}\right) \in \Omega \times \bar{\Lambda}, O^{\prime} \supseteq O, \lambda_{O^{\prime}} \prec \lambda_{O}$, and $S\left(\lambda_{O}\right) \tilde{\epsilon} O$.
Thus, $S\left(\lambda_{O}\right) \tilde{\epsilon} O^{\prime}$. So, $\left\{S\left(\lambda_{O}\right):\left(O, \lambda_{O}\right) \in \Omega \times \bar{\Lambda}\right\}$ converges to $\xi$. That is, there is a subnet of $S$ converging to $\xi$.

Theorem 8. Let $F_{A} \in F S(U, E)$. Then, $F_{A}$ is compact if and only if any net whose element is quasi-coincident with $F_{A}$ has a subnet whose limit is quasi-coincident with $F_{A}$.

Proof (Necessity). Let $F_{A}$ be a compact fuzzy soft set and $S=\{S(\lambda): \lambda \in \Lambda\}$ a net whose element is quasi-coincident with $F_{A}$. To prove the Necessity, it must only be shown that $S$ has a subnet whose limit is quasi-coincident with $F_{A}$. Suppose it is not true, then for any point $\xi$ which is quasicoincident with $F_{A}$, any subnet of $S$ does not take $\xi$ as its limit. Therefore, by Lemma 1 , there exists $O_{\xi} \in \mathrm{A}(\xi)$ and $\lambda_{\xi} \in \Lambda$ such that when $\lambda_{\xi} \prec \lambda \in \Lambda$, and

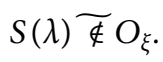

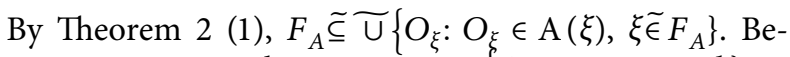
cause $F_{A}$ is compact, there exists $\Psi=\left\{O_{\xi_{i}}: i=1,2 \ldots, k\right\} \subseteq \Omega$ such that $F_{A} \widetilde{\subseteq} \cup_{i=1}^{k} O_{\xi}$. For each $i=1,2, \ldots, k$, by (5), there exists $\lambda_{\xi_{i}} \in \Lambda, S(\lambda) \notin O_{\xi_{i}}$ whenever $\lambda_{\xi_{i}}<\lambda \in \Lambda$. Because $\Lambda$ is a directed set, there exists $\lambda_{0} \in \Lambda$ such that $\lambda_{\xi_{i}} \prec \lambda_{0}$ $(i=1,2, \ldots, k)$. It follows that $S\left(\lambda_{0}\right) \approx O_{\xi_{i}}(i=1,2, \ldots, k)$. Then, $S\left(\lambda_{0}\right) \notin F_{A}$. This is in contradiction with the supposition that $S=\{S(\lambda): \lambda \in \Lambda\}$ is a net whose element is quasi-coincident with $F_{A}$. Thus, $S$ has a subnet whose limit is quasi-coincident with $F_{A}$.

(Sufficiency) Let $\Theta=\left\{O_{i}: i \in \Gamma\right\}$ be an open cover of $F_{A}$, $\Lambda=\{\lambda: \lambda$ is a finite subset of $\Gamma\}$. Under the usual inclusion " $\subseteq$ " relation, $\Lambda$ forms a directed set. Suppose that $F_{A}$ is not compact, then for any $\lambda \in \Lambda, F_{A} \widetilde{\subseteq} \cup O_{i}$. Then, by Theorem 2 (1), there is a point $S(\lambda) \tilde{\epsilon} F_{A}$; however,

$$
S(\lambda) \widetilde{\Psi} \cup_{i \in \lambda} O_{i}, \quad \forall \lambda \in \Lambda
$$

Thus, a net $S=\{S(\lambda): \lambda \in \Lambda\}$ is obtained whose element is always quasi-coincident with $F_{A}$; however, $S(\lambda) \notin \underset{i \in \lambda}{\forall} O_{i}$,
$\forall \lambda \in \Lambda$.

Under the assumption, there is a subnet $T=\{T(\omega): \omega \in \Omega\}$ of $S$ such that $\lim T(\omega)=\xi_{0}, \tilde{\epsilon} F_{A}$. Because $F_{A} \widetilde{\subseteq} \cup O_{i \in \Gamma}$ and due to Theorem 2 (3), there exists $i_{0} \in \Gamma$ such that $\xi_{0} \tilde{\epsilon} O_{i_{0}}$. So, there exists $\omega_{1} \in \Omega$ when $\omega_{1} \prec \omega \in \Omega$ :

$$
T(\omega) \tilde{\epsilon} O_{i_{0}} .
$$

Because $T=\{T(\omega): \omega \in \Omega\}$ is a subnet of $S=\{S(\lambda): \lambda$ $\in \Lambda\}$, there exists a mapping $\varphi$ from $\Omega$ to $\Lambda$. For the above $i_{0}$, $\left\{i_{0}\right\} \in \Lambda$. Hence, there exists $\omega_{2} \in \Omega$ such that $\left\{i_{0}\right\}<\varphi\left(\omega_{2}\right)$. Subsequently, when $\omega_{2} \prec \omega \in \Omega$, the following is obtained: $\left\{i_{0}\right\} \prec \varphi(\omega)$, that is, $i_{0} \in \varphi(\omega)$. From (6), $S(\varphi(\omega)) \notin O_{i_{0}}$, that is,

$$
T(\omega) \rightleftarrows O_{i_{0}} .
$$

Let $\omega \in \Omega$ with $\omega_{1} \prec \omega$ and $\omega_{2} \prec \omega$; then, both (7) and (8) have a contradiction. Therefore, $F_{A}$ is compact. 


\section{Conclusions}

In this paper, we have obtained some important theorems by means of the convergence of a fuzzy soft net. The obtained results demonstrate that the existing net is a powerful tool for studying fuzzy soft topological spaces. It is prospective that fuzzy soft nets will play important roles in characterizing some other properties of fuzzy soft topological spaces.

\section{Data Availability}

No data were used to support this study.

\section{Conflicts of Interest}

The authors declare that they have no conflicts of interest.

\section{Authors' Contributions}

Jianrong Wu conceptualized the study. Rui Gao was responsible for formal analysis. Jianrong $\mathrm{Wu}$ acquired funding. Rui Gao investigated the study. Jianrong $\mathrm{Wu}$ was responsible for methodology. Rui Gao wrote the original draft. Jianrong $\mathrm{Wu}$ wrote, reviewed, and edited the manuscript. All authors have read and agreed to the published version of the manuscript.

\section{Acknowledgments}

This work was supported by the National Natural Science Foundation of China (11971343) and the Postgraduate Research and Practice Innovation Program of Jiangsu Province (KYCX19_2015).

\section{References}

[1] P. K. Maji, R. Biswas, and A. R. Roy, "Fuzzy soft sets," Journal of Fuzzy Mathematics, vol. 9, pp. 589-602, 2001.

[2] L. A. Zadeh, "Fuzzy sets," Information and Control, vol. 8, no. 3, pp. 338-353, 1965.

[3] D. Molodtsov, "Soft set theory-First results," Computers \& Mathematics with Applications, vol. 37, no. 4-5, pp. 19-31, 1999.

[4] S. Alkhazaleh, A. R. Salleh, and N. Hassan, "Fuzzy parameterized interval-valued fuzzy soft set," Applied Mathematical Sciences, vol. 5, pp. 3335-3346, 2011.

[5] A. Aygünoğlu and H. Aygün, "Introduction to fuzzy soft groups," Computers \& Mathematics with Applications, vol. 58, pp. 1279-1286, 2009.

[6] F. Feng, Y. Li, and V. Leoreanu-Fotea, "Application of level soft sets in decision making based on interval-valued fuzzy soft sets," Computers \& Mathematics with Applications, vol. 60, no. 6, pp. 1756-1767, 2010.

[7] R. Gao and J. R. Wu, "A net with applications for continuity in a fuzzy soft topological space," Mathematical Problems in Engineering, vol. 2020, Article ID 9098410, 7 pages, 2020.

[8] Z. Kong, L. Gao, and L. Wang, "Comment on "A fuzzy soft set theoretic approach to decision making problems", "Journal of Computational and Applied Mathematics, vol. 223, no. 2, pp. 540-542, 2009.

[9] A. R. Roy and P. K. Maji, "A fuzzy soft set theoretic approach to decision making problems," Journal of Computational and Applied Mathematics, vol. 203, no. 2, pp. 412-418, 2007.
[10] J. Wang, Y. Hu, F. Xiao, X. Deng, and Y. Deng, “A novel method to use fuzzy soft sets in decision making based on ambiguity measure and dempster-shafer theory of evidence: an application in medical diagnosis," Artificial Intelligence in Medicine, vol. 69, pp. 1-11, 2016.

[11] Z. Xiao, K. Gong, and Y. Zou, "A combined forecasting approach based on fuzzy soft sets," Journal of Computational and Applied Mathematics, vol. 228, no. 1, pp. 326-333, 2009.

[12] N. X. Xie, G. Q. Wen, and Z. W. Li, "A method for fuzzy soft sets in decision making based on grey relational analysis and d-s theory of evidence: application to medical diagnosis," Computational and Mathematical Methods in Medicine, vol. 2014, Article ID 581316, 12 pages, 2014.

[13] Z. Zhang and S. Zhang, "A novel approach to multi attribute group decision making based on trapezoidal interval type-2 fuzzy soft sets," Applied Mathematical Modelling, vol. 37, no. 7, pp. 4948-4971, 2013.

[14] A. Aygünoğlu, E. Aydoğdu, and H. Aygün, "Fuzzy soft metric and fuzzifying soft topology induced by fuzzy soft metric," Filomat, vol. 33, pp. 645-653, 2019.

[15] S. El-Shiekh and S. El-Sayedp, " $\gamma$-Operation \& decomposition of some forms of fuzzy soft mappings on fuzzy soft ideal topological spaces," Filomat, vol. 34, 2020.

[16] C. Gunduz (Aras) and S. Bayramov, "Some results on fuzzy soft topological spaces," Mathematical Problems in Engineering, vol. 2013, Article ID 835308, 10 pages, 2013.

[17] I. Ibedou and S. E. Abbas, "Fuzzy soft filter convergence," Filomat, vol. 32, no. 9, pp. 3325-3336, 2018.

[18] A. Kandil, O. A. El-Tantawy, S. A. El-Sheikh, and S. S. S. ElSayed, "Fuzzy soft connected sets in fuzzy soft topological spaces II," Journal of the Egyptian Mathematical Society, vol. 25, no. 2, pp. 171-177, 2017.

[19] J. Mahanta and P. K. Das, "Results on fuzzy soft topological spaces," 2012, http://arxiv.org/abs/1203.0634v1.

[20] J. S. Ping, T. Wu, and C. Z. Yang, "Sum spaces in fuzzy soft topological spaces," Fuzzy Systems and Mathematics, vol. 28, pp. 69-73, 2014.

[21] S. Roy and T. K. Samanta, "An introduction to open and closed sets on fuzzy soft topological spaces," Annals of Fuzzy Mathematics and Informatics, vol. 6, pp. 425-431, 2013.

[22] S. Roy and T. K. Samanta, "A note on fuzzy soft topological spaces," Annals of Fuzzy Mathematics and Informatics, vol. 3, pp. 305-311, 2012.

[23] T. Simsekler and S. Yuksel, "Fuzzy soft topological spaces," Annals of Fuzzy Mathematics and Informatics, vol. 5, pp. 87-96, 2013.

[24] B. Tanay and M. B. Kandemir, "Topological structure of fuzzy soft sets," Computers \& Mathematics with Applications, vol. 61, no. 10, pp. 2952-2957, 2011. 